JOANNA DRYNDA

\title{
Grenzgänge. Zu (männlichen) Ausbrüchen aus emotionaler Routine im Romanwerk von Stephan Thome
}

Routine tötet das Leben und vertieft den Fluch, geboren zu sein.

(Emil Cioran)

\begin{abstract}
Artykuł poświęcony jest dwóm powieściom niemieckiego pisarza Stephana Thome: Grenzgang (2009) oraz Fliehkräfte (2012). Punktem ciężkości tych po części melancholijnych, po części dowcipnych narracji są desperackie, mniej lub bardziej udane próby uwolnienia się bohaterów z emocjonalnej rutyny, która po upadku habitualnych norm męskości przejawia się jako prowizoryczny schron dla ,udręczonego ego " męskiego podmiotu. Punktem wyjścia rozważań są prace socjologiczne i kulturoznawcze, które obalają tezę, jakoby spektrum męskich emocji ograniczało się do tłumienia uczuć i dyscyplinowania patologicznych namiętności.
\end{abstract}

Der Aufsatz ist zwei Romanen des deutschen Schriftstellers Stephan Thome gewidmet. Den Kristallisationspunkt der teils melancholischen, teils witzig pointierten Narrationen bilden sowohl in Grenzgang (2009) als auch in Fliehkräfte (2012) die verzweifelten, mehr oder minder geglückten Versuche männlicher Protagonisten, sich aus emotionaler Routine zu befreien, die in Zeiten des Abgesangs auf habituelle Männlichkeitsnormen, in unterschiedlicher Ausprägung dem männlichen Subjekt als ein notdürftiger Schutzbau für , sein geschundenes Ego ' dient. Den Ausgangspunkt bilden die soziokulturell angelegten Studien, die die These widerlegen, dass das Spektrum männlicher Emotionen sich auf die Unterdrückung der Gefühle und die Disziplinierung pathologischer Leidenschaften beschränke.

The article is devoted to two novels of the German writer Stephen Thome: Grenzgang (2009) and Fliehkräfte (2012). The essential element of those partly melancholic, partly witty narratives are desperate, more or less successful attempts to free the literary heroes from emotional routine which - after the fall of habitual norms of mascu- 
Joanna Drynda

linity - manifests itself as a makeshift shelter for the ,plagued ego' of the male subject. The point of departure in relation to those deliberations are sociological and cultural works which debunk the thesis that the spectrum of male emotions limits itself to suppressing emotions as well as to disciplining pathological desires.

\section{1. ,Ich fühle, also bin ich.“ (DAMASIO 2000)}

Das Diktum des amerikanischen Neurobiologen Antonio R. Damasio wird gern Studien vorangestellt, die der seit geraumer Zeit anhaltenden Renaissance des Gefühls nachgehen. Im breiten Spektrum der Forschungsperspektiven, das von Neurowissenschaften über Philosophie bis hin zu Kultur- und Sozialwissenschaften reicht, erblickt man Anzeichen für den Emotional Turn $^{1}$, der auf einen ,erheblichen Nachholbedarf “2 zurückgeführt wird. Der Grundtenor heterogener Theorien besteht nämlich in der Kritik des in der westlichen Moderne dominanten Rationalitätsparadigmas, das die Erlebnisqualität der Emotionen marginalisierte und Gefühle als etwas Vernunftwidriges abstempelte. Hat man sich einstweilen weitgehend darauf geeinigt, Emotionen seien nicht ,das Andere' des Verstandes, sondern verfügen über eine eigene kognitive Dimension (BORUTTA / VERHEYN 2010:23) ${ }^{3}$, so werden trotz dieser Grunderkenntnis gewichtige Unterschiede sichtbar, und zwar bereits in der Frage, wie der Forschungsgegenstand - Emotion/Gefühl ${ }^{4}$ trennscharf abzugrenzen wäre. Bei der Fülle neuer Einsichten und teils unvereinbarer Definitionsversuche geht nicht nur der Überblick leicht verloren, vielmehr scheint die Möglichkeit, Emotionen eindeutig zu fassen, im Zuge der Differenzierung der Emotionsforschung sogar abzunehmen (vgl. BORUTTA / VERHEYN 2010:17). Wenn von einem transdisziplinären Konsens ge-

1 Thomas Anz: Emotional Turn? Beobachtungen zur Gefühlsforschung. http:// www.literaturkritik.de/public/rezension.php?rez_id=10267 (27.05.2014).

2 Vgl. Thomas Anz: Die Wissenschaften und die Gefühle. Skizzen und Literaturhinweise. http://www.literaturkritik.de/public/rezension.php?rez_id=3277 (27.05.2014).

3 Zu philosophischen Debatten über Emotionen als Kognitionen vgl. BERNINGER / DÖRING (2009:141-147).

4 „Daß Emotionen ,keine Gefühle‘ sind, ist heute unter Philosophen beinahe schon ein Gemeinplatz", so Robert C. Roberts (2009:172); bei Emotionen handele es sich um einen Terminus technicus für Gefühle im engeren Sinne, die sich gegenüber ,nichtemotionalen Gefühlen“ dadurch auszeichnen würden, „daß sie auf etwas in der Welt gerichtet sind und es in bestimmter Weise seiend repräsentieren." (vgl. DÖRING 2009:14) 
sprochen werden kann, dann bestehe er allenfalls darüber, dass es sich bei Emotionen um hochkomplexe Phänomene handele, die - je nach der verbindlichen Optik - weiter spezifiziert würden.

In soziokulturell angelegten Studien werden Emotionen und ihre Kultivierung in einen sozialen Kontext eingebettet. Bei der Analyse ihrer Durchformung gilt die besondere Aufmerksamkeit der Kategorie Geschlecht, was auf die signifikante, geschlechtskonnotierte Geschichte des Emotionsbegriffs zurückgeht (vgl. FREVERT 2010:309f.). Die Zuschreibung von Weiblichkeit und Emotionalität sowie Männlichkeit und Rationalität hatte zur Folge, dass männliche Gefühle meist negativ thematisiert wurden: als „Unterdrückung, Disziplinierung oder verhängnisvolle Entfesselung pathologischer Leidenschaften“ (BORUTTA / VERHEYEN 2010:13). Die These von einer defizitären maskulinen Emotionalität stößt derzeit auf heftige Kritik und wird von Forschern widerlegt, die das ,gendering ' von Vernunft und Gefühl als einen historisch wandelbaren Prozess auffassen und die affektiven Formungen des Subjekts in Zusammenhang mit Wandlungen der Geschlechterordnung betrachten. Wie ANDREAS RECKWITZ (2010:73) betont, stelle die Genealogie der Affekte und Geschlechter ,eine diskontinuierliche Transformation mehrerer einander widersprechender und miteinander konfligierender Formationen dar, die in kein lineares Muster zu pressen“ sei. Daraus folgt zweierlei: zum einen, dass das männliche - wie das weibliche - Subjekt im Widerstreit zwischen , gendering/degendering ' und Emotionalisierung/Entemotionalisierung ständig (um)modelliert wird; und zum anderen, dass gewaltige Kulturkonflikte auch die emotionale Subjektformung in der Mikroskala eines einzelnen Lebenskonzeptes beeinflussen. Wenn also Emotionen der integrale Bestandteil des männlichen Subjektkonstrukts sind, stellt sich die grundsätzliche Frage, ,welche Emotionen der vermeintlichen Rationalität von Männlichkeit zugrunde liegen“ (SCHOLZ 2010:206). Einen Erklärungsrahmen dafür eröffnet die Literatur, da sie dem narrativen Charakter der Emotionsphänomene entgegenkommt. In dem Spiel mit fiktiven Identitäten zeige sich die Wichtigkeit der narrativen Struktur im Verstehen und in der Interpretation emotionaler Erfahrungen, so der Philosoph Peter Goldie. Der narrative Zusammenhang gewinnt seiner Meinung nach besonders dann an Relevanz, wenn ein hoher Grad an Sensitivität gegenüber Einzelheiten der emotionalen Erfahrung vorhanden sei, wenn ,zwei oder mehrere Emotionen oft zur gleichen Zeit erlebt werden und qualitativ ähnliche Gefühle mit einschließen“ (GOLDIE 2009:394), oder aber, wenn sich „,die verschiedenen ,gegensätzlichen' Eigenschaften von Emotionen in vielfältiger Weise überkreuzen und eine klinisch reine begriffliche Schematisierung vereiteln“ (ROBERTS 2009a:271). 
Joanna Drynda

Für verworrene emotionale Schlüsselszenarien hat der deutsche Romancier Stephan Thome (Jahrgang 1972) ein besonderes Faible. Sowohl in dem vielbeachteten Debüt Grenzgang (2009) als auch in dem zweiten Roman Fliehkräfte (2012) - beide standen auf der Shortliste zum Deutschen Buchpreis thematisiert er die moderne Erfahrungs- und Empfindungswelt und liefert einen Einblick in den Gefühlshaushalt des männlichen Subjekts zu Beginn des 21. Jahrhunderts. Die Texte bündeln gesellschaftliche Codes und Spannungen in einer Subjektgeschichte, wobei der Blick auf die individuelle Geschichte gerichtet ist, die einen Mann zu dem machte, wie er ist, oder, um auf Damasios Bonmot zurückzukommen, wie er nicht ist, weil er (scheinbar) nichts fühlt.

\section{2. ,ich bin nicht immer so gewesen“": Akteure, Emotionen, Routinen}

„Wissen Sie, ich bin nicht immer so gewesen. Ich muss mich erst wieder umgewöhnen." (THOME 2009:186) Mit diesen Worten bringt eine Figur in Grenzgang das Hauptmotiv beider Romane - den Prozess der Befreiung aus emotionaler Routine - auf den Punkt. Mit Raffinement schildert Thome missglückte Lebensentwürfe der Protagonisten, die auf dem Weg durch ihre Biographie Schritt für Schritt hinter eigenen Träumen zurückbleiben. Vor dem Geständnis, kläglich versagt zu haben, retten sie sich dadurch, dass sie gefühlsmäßig erstarren. Wenngleich sich beide männlichen Hauptfiguren in einer beruflich oder familiär bedingten emotionalen Notlage befinden, ist sowohl der Grund als auch der Zeitpunkt der inneren Lähmung ein anderer.

Hartmut Hainbach, ein Endfünfziger aus Fliehkräfte erreichte das, was Thomas Weidmann, dem um eine Dekade jüngeren Protagonisten in Grenzgang, nicht gelang - sich von einem provinziellen Herkunftsmilieu zu emanzipieren und ordentlicher Professor zu werden, wenn auch das Warten auf die Berufung nach Berlin vergeblich bleiben sollte. Der Berliner Traum geht für den Historiker Weidmann nicht in Erfüllung, weil die Habilitation, an der er jahrelang feilt, nicht angenommen wird. Aus diesem Grund bricht er seine Zelte ab und kehrt in die Heimatstadt zurück, der er entschieden den Rücken wenden wollte. Dort übermannt ein provinzieller Tiefschlaf den enttäuschten Deserteur, der am Gymnasium unterrichtet und dem Klischeebild eines alleinstehenden Studienrates immer ähnlicher wird. Im Unterschied zu der mit wenigen Liebesaffären geschmückten Singleexistenz Weidmanns führt Hainbach ein glückliches Familienleben. Die Idylle des bürgerlichen Glücks bricht allerdings zusammen, als die einzige Tochter das Haus verlässt, um in 
Spanien zu studieren, und die Ehefrau nach Berlin geht, wo sie als Assistentin eines exzentrischen Theatermachers, ihres Ex-Geliebten, beruflich Fuß zu fassen versucht. Die sorgfältig geplanten Treffen an den Wochenenden täuschen den Mann über das entstandene Sinnvakuum kaum hinweg: „Seitdem lebte er mit dem Gefühl, seiner Frau hinterherzusehen. Hatte sich in Stress und Einsamkeit ergeben und nicht gemerkt, wie groß sein Verlangen geworden war, selbst vorauszufahren.“ (THOME 2012:146) Zudem kommt es ihm vor, die emotionale Integrität eingebüßt zu haben: „Leben im Diskontinuum, er weiß nicht, wie er sich fühlt.“ (THOME 2012:456) Weidmann hingegen, der sich im Einerlei des schulischen Alltags wie im Alleinsein nach außen hin gut eingerichtet hat, überspielt ironisch seine emotionale Abstumpfung: „Abwesenheit von Gefühlen, stellte er fest, ist auch ein Gefühl.“ (THOME 2009:39) Beide Männer leben in einer emotionalen Lethargie konserviert, doch während die Routine von Weidmann nach der beruflichen Niederlage und der damit verbundenen emotionalen Kosten mehr oder weniger bewusst als Refugium gesucht wird, merkt Hainbach nicht einmal, wann sie sich in sein Leben einschleicht, um die Fundamente der Ehe zu unterspülen, so dass erst das Fortgehen der Frau ihm Trugbilder vor Augen führt, die ihn jahrelang narrten. Für die zwei Grundbefindlichkeiten sowie Arten, mit emotionalen Herausforderungen umzugehen, findet Thome zwei Inszenierungsformen, die trotz gewisser Analogien in der Roman- und Figurenkonstellation, nicht auf einen Nenner zu bringen sind.

Das narrative Gerüst beider Romane ist der Montagetechnik verpflichtet der Autor springt durch die Zeiten, schildert punktuelle Ereignisse aus einem Zeitraum von mehreren Dekaden, die Lücken dazwischen werden in Rückblenden rekonstruiert. Als eine Konstruktionsklammer fungiert in Grenzgang das titelgebende Volksfest, das alle sieben Jahre den Alltag der Provinzstadt auf den Kopf stellt. Wird in Fliehkräfte ebenfalls in großen Zeitsprüngen erzählt, so folgt man der Hauptfigur im Rhythmus einer Reise, die aus Bonn über Frankreich und Spanien nach Portugal führt. Die Reisestationen wechseln mit chronologisch geschnittenen Reminiszenzen: an die Studienzeit in den USA, an das Berliner Künstlermilieu, an die Familie in Deutschland und Portugal, woher Hainbachs Ehefrau stammt. Die männlichen Figuren spiegeln sich konsequent in den weiblichen wider, wiewohl auf verschiedene Weise. Dem dynamischen Modell der Reise entspricht in Fliehkräfte die Vielzahl weiblicher Pendants, die Hainbach aus der emotionalen Reserve locken. Mit der Eintönigkeit des wiederkehrenden Festes korrespondiert in Grenzgang dagegen die statische Spiegelstruktur: Parallel zu Weidmanns Schicksal wird die Lebensgeschichte von Kerstin Werner dargestellt, einer 
Joanna Drynda

geschiedenen Mittvierzigerin, zu deren ohnehin beträchtlichem innerem Gefühlschaos der pubertierende Sohn ebenso beiträgt wie die pflegebedürftige, demenzkranke Mutter.

\section{3. „Hektisch versucht sein Verstand, die Situation zu erfassen“ (THOME 2012:415)}

Die auf der Figurenebene arrangierte Konfrontation von Mann und Frau ruft unweigerlich geschlechtsspezifisch zugeordnete Assoziationen von Emotionalität und Rationalität hervor. In Fliehkräfte sticht diese Zuschreibung insofern ins Auge, als die männliche Hauptfigur die Rationalität geradezu zu verkörpern scheint. Der studierte Philosoph Hainbach präsentiert sich als der Prototyp eines Denkers, der den Gelehrtenhabitus selbst in seinen vier Wänden wahrt. Das hartnäckige Bestehen auf einer nüchternen Einschätzung der Lage wie auch der Hang, gründlich ins letzte Detail zu gehen, erregen das Spottbedürfnis der Tochter, die vor einem zu fassenden Entschluss fragt, ob „er bereits in den gewiss mehrstufigen Entscheidungsprozess eingetreten“ (THOME 2012:256) sei. Unter fehlender Spontaneität und dem Glauben an rationale Argumente, mit denen einmal getroffene Entscheidungen gerechtfertigt werden, leidet die Ehefrau - eine um einiges jüngere, temperamentvolle Südländerin mit künstlerischen Ambitionen. Zwar unterstützt sie den Mann bei seinem Klettern auf der universitären Karriereleiter und versteht dessen Stolz auf das Erreichte, ist aber nicht bereit, für den Wohlstand und die Stabilität eines geregelten Lebens, was dem Mann als die Krönung seiner beruflichen Laufbahn erscheint, auf Dauer mit Stagnation zu bezahlen. Auf den ersten Blick wirkt das Paar in Fliehkräfte in den Eherollen gleichermaßen stereotyp wie unzeitgemäß: sie - eine Hausfrau, die mehr aus Langeweile als aus finanzieller Not Portugiesischkurse leitet; er - ein Alleinverdiener, der arbeitet, um ihre Langeweile zu finanzieren. Das Bild, das der Mann abgibt, ist kennzeichnend für die ,archetypische, traditionelle Männlichkeit - eine Schutz bietende Männlichkeit“ (ILLOUZ 2013:60), die generell mit der Ungleichheit in der Mann-Frau-Relation einhergeht. Doch verfüge das asymmetrische Verhältnis von beschützendem Mann und abhängiger Frau, so Eva Illouz, zweifellos über angenehme Aspekte. Zum einen erzeuge die von der Macht- in ein Beschützerverhältnis umgewandelte Ungleichheit eine ,natürliche" Abhängigkeit und einen starken emotionalen ,Klebstoff', zum anderen gebe es Klarheit bezüglich der Rollen, die nicht erst ausgehandelt werden müssten, was ,zu spontaneren, unmittelbareren und weniger verkopften Ge- 
fühlen“ beitrage (ILLOUZ 2013:58f.). Indem der Mann bewährten Drehbüchern folgt, fühlt er sich in der Beschützerrolle gut aufgehoben, da sie weder Ängste oder Unsicherheit auslöst, noch von ihm große Taten abverlangt. Freilich basiert die dergestalt affektiv aufgeladene Beziehung auf einer projektiven Realitätsverkennung. Ohne zu reflektieren, inwiefern das emotionale Beziehungsgerüst überhaupt tragfähig ist, überträgt der Mann sein Wohlgefühl auf die Frau, bis sie ihm an den Kopf wirft: „Unser Leben ist die Parodie unserer Träume [...].“ (THOME 2012:455)

Die Frau will sich nicht scheiden lassen, geschweige denn dem Mann weh tun, sondern die Chance ergreifen, nach zwanzig Jahren der hausfraulichen Öde zu entkommen. Hilflos und resigniert nimmt er ihren Umzug nach Berlin hin, doch bald erfasst ihn Panik, dass alles dem Ende zusteuern könnte: ,Ihn haben die vergangenen zwei Jahre gelehrt, dass Liebe ein schwaches Argument sein kann. Schwächer als einsame Nächte, die Frustration über ihr abgeschaltetes Handy oder das merkwürdige Gefühl beim Betreten von Marias Wohnung." (THOME 2012:40) Da Maria Hainbachs Ein und Alles ist, kann er der physischen Distanz nichts abgewinnen, im Gegenteil, die räumliche Entfernung wird mit der emotionalen gleichgesetzt. Umgekehrt nimmt die Frau Merkmale männlicher Autonomie an: Sie kämpft entschieden um ihre Selbstbestimmung, davon überzeugt, die Ehe könne von ihrer Selbstständigkeit, und sei diese andernorts unter Beweis gestellt, nur profitieren. Indes sie in der neuen Umgebung aufblüht, verzehrt sich der Mann vor Sehnsucht nach ihr.

Es fällt auf, dass es kaum möglich ist, den Protagonisten auf eine Reihe eindeutiger Merkmale festzulegen, denn so sehr Rationalität, Verantwortung und sozial überlegene Position mit klassischen Männlichkeitsbildern harmonieren, so wenig passt darin seine - traditionell feminin konnotierte - „Sehnsucht nach einer emotionalen Symbiose“ (ILLOUZ 2013:45). Dass das Wesen der symbiotischen Ehebeziehung vorwiegend darin bestand, die Gefahr eines Gefühlsaustausches zu vermeiden, leuchtet dem Mann ein, sobald er, im Bonner Haus zurückgelassen, in den „Zustand einer ständigen Übertreibung“ (THOME 2012:117) gerät. Der ihn überwältigende Gefühlswirrwarr wird umso beträchtlicher, als sich zu dem längst Aufgestauten noch Eifersucht, Einsamkeit, Leere, Müdigkeit, Ratlosigkeit, Verzweiflung und Wut gesellen. Ohne unterscheiden zu können, welche der Emotionen dominiert, gestattet er sich, die Gefühle nicht zu unterdrücken. Dabei kommt die Einsicht, dass das Wunschbild eines ausgeglichenen Beisammenseins direkt in eine emotionale Sackgasse führt: „Die Fähigkeit, ohne großen emotionalen Aufwand zu lie- 
ben, sei das Geheimnis einer langen (im mutmaßlichen Gegensatz zur bloß glücklichen) Ehe.“ (THOME 2012:41) Dennoch ist seine Haltung ambivalent, als sich die Gelegenheit ergibt, das Heikle an der Beziehung auszudiskutieren: ,Streitvermeidung als oberstes Prinzip einer Ehe garantiert nicht Harmonie, sondern Stagnation. Wahrscheinlich muss man große Angst vor etwas anderem haben, um darin das kleinere Übel zu sehen." (THOME 2012:39)

Für die Schilderung des Ausbruchs aus der Stagnation wird das filmische Paradigma eines Roadmovies literarisch adaptiert. Entgegen den Gewohnheiten entscheidet sich der Mann spontan für die Reise ohne einen festen Zeitplan, die unruhige Wanderschaft folgt einer linearen Logik des klassischen Musters, die auf Überwindung eines Hindernisses abzielt. Die Entscheidung, die unterwegs zu fällen ist, betrifft das Arbeitsangebot eines kleinen Berliner Verlags und das damit verbundene Risiko eines Neuanfangs: Um gemeinsam mit Marie in Berlin leben zu können, müsste die Bonner Professur in die Waagschale geworfen werden und somit außer der finanziellen Stabilität auch der Anspruch auf die Beamtenrente. Mithin wäre der Preis für die emotionale Sicherheit mit dem Autonomieverlust verbunden, und dies ohne Sicherheit, ob die große Aufopferung von der Frau nicht abgelehnt wird. Der narrativen Matrix entsprechend, nach dem Motto „Es gibt nichts Demaskierenderes als die Maske selbst“ (THOME 2012:127), wird Hainbachs Selbstbild auf jeder Reisestation auf die Probe gestellt, bis er den Charakter der Spannung zwischen Autonomie und Anerkennung begreift, welche laut Illouz als ein Paradox unverbrüchlich in die gegenwärtige Liebesordnung eingeschrieben sei. Die Spannung ergibt sich daraus, dass sich die Anerkennung innerhalb einer Dynamik vollzieht, in der es die eigene Autonomie stets zur Schau zu stellen gilt. Da die Autonomie durch eine sorgfältige Überwachung und sogar Verweigerung von Anerkennung erreicht wird, tut sich das Bedürfnis kund, ,den Ausdruck von Gefühlen (die Anerkennung eines anderen) unter Beobachtung zu behalten, um die eigene Position in einer Beziehung nicht zu schwächen“ (ILlOUZ 2012:243f.). Betrachtet man vor diesem Hintergrund Hainbachs Beschützerdrang, so wird deutlich, dass dieser im Grunde darauf hinausläuft, die eigene Autonomie, ergo das eigene Selbstwertgefühl zu schützen. Wie schwer es dem Mann hingegen fällt, fern von Marias anerkennenden Augen das Selbstbild aufrecht zu erhalten, beweisen Äußerungen wie diese: „Er kannte das Gefühl, obwohl er es lange nicht empfunden hatte: $\mathrm{Zu}$ Hause sein zu wollen und nicht zu wissen wo. Bloß zu spüren, wie es wäre.“ (THOME 2012:34) Dass in der qualitativ neuen Realität das Selbstverständnis als Mann neu definiert werden muss, wird im Gespräch mit einem Freund greifbar. Mit verstohlenem Neid beschreibt der Freund seinen Vater wie 
folgt: „Er hat sein Leben getragen, wie einen maßgeschneiderten Anzug. Oder umgekehrt, das Leben ihn, keine Ahnung. Jedenfalls war er genauso, wie er sein musste.“ (THOME 2012:198) Solche Gewissheit, als Mann „seinen Ort [zu] haben“, oder zumindest auf dem rechten Weg dorthin zu sein, hat die nächste Generation nicht. Vielmehr irren die Söhne von einem Konzept zum nächsten, um bitter zu erfahren, wie ihr Selbstbild immerfort wie ein Kartenhaus in sich zusammenfällt. So gesehen wird mit dem Reisemotiv die Schwellensituation eines männlichen Subjekts konzeptuell erfasst, das Unterwegssein zwischen Nicht-Ganz-Hier und Nicht-Ganz-Da.

Was den Roman daran hindert, in eine monotone Schilderung des Selbstfindungsprozesses abzugleiten, sind die Rückblenden innerhalb der Narration, die Hainbachs Werdegang sichtbar machen, seine innere Reise zu jenem Mann, der ihm eines Tages im Wege stehen wird. Freilich ahnt der Protagonist, dass es sich bei dem Konzept von Männlichkeit um eine ebenso dynamische wie krisenanfällige Konstruktion handelt: „Es könnte bloß sein, dass menschliches Leben nicht nur ein Mal beginnt. Weil es aus Phasen besteht, die alle ihren eigenen Beginn haben." (THOME 2012:39) Durch die exakte zeitliche Verortung der sechs Erinnerungssequenzen (von 1973 bis 1998) umreißt Thome Konturen einer emotionalen Grammatik gegenwärtiger Männlichkeit. Diese konstituiere sich, so SYLKA SCHOLZ (2010:206) in Anlehnung an soziologische Studien von Robert W. Connell, Pierre Bourdieu und Michael Meuser, in einer doppelten Relation: in Abgrenzung zu Weiblichkeit sowie zu anderen Männlichkeiten, die sich flexibel mit anderen Kategorien (Generation, Klasse, Milieu etc.) verknüpfen.

Den weiblichen Romanfiguren fällt es trotz der Generationsunterschiede oder des Herkunftsmilieus insgesamt leichter, den Gefühlen freien Lauf zu lassen, ob verbal oder nonverbal: „Apropos Mutter: Mach dich auf Tränen gefasst. Deine Rückkehr ist der emotionale Höhepunkt des Jahres, wenn nicht des Jahrzehnts. “ (THOME 2012:110) Im Vergleich dazu erlebt Hainbach am väterlichen Vorbild die Männlichkeit als eine entemotionalisierte Distanziertheit in der Verhaltensweise. Der Vater huldigt dem proletarischen Männlichkeitsideal der Nachkriegszeit, das Wortkargheit, die Betonung physischer Kraft sowie Charaktereigenschaften wie Disziplin und Strenge in sich vereint (vgl. ScHOLZ 2010:210). Den zentralen Bezugspunkt seiner Existenz bildet außer der Erwerbsarbeit die erbrachte Leistung: „Leben und arbeiten war dasselbe für ihn.“ (THOME 2012:199) Familiäre Bindungen sind zwar von Bedeutung, der Umgang mit den Nächsten zeichnet sich aber durch schroffe Kälte aus; Frau und Tochter haben zu gehorchen, der Stammhalter wird fallweise ver- 
prügelt. Für den ehrgeizigen Arbeitersohn bedeutet das Stipendium in den USA eine doppelte Rettung: einerseits vor der Kälte des Vaters oder vor der tiefen Scham angesichts des Hauses ohne Bücher, andererseits vor ideologischen Fragen, für die der unsichere Autodidakt keine Antennen hat und mit denen er zu Beginn des Studiums in Berlin konfrontiert wird. Anfang der 1970er Jahre erscheint das Desinteresse an Politik in engagierten Studentenkreisen suspekt, was den ohnehin schon gehemmten jungen Mann zusehends paralysiert und zum Außenseiter macht. Entmutigt bleibt er aus dem Beziehungsnetzwerk der Revolutionäre ausgeschlossen, zumal der „maskulinautoritäre Charakter derjenigen Gruppierungen, die sich selbst zum antiautoritären Flügel zählten“, so ARIBERT REIMANN (2010:231) über den männerbündnischen Charakter kulturrevolutionärer Subkulturen der 1960er und 1970er Jahre, Hainbach insofern abstößt, als er sich abermals bevormundet fühlt. Somit sieht er sich einer Welt entgegengesetzt, gegen die man sich durch eine sachliche Haltung sowie durch die Verkapselung der Gefühle innerlich zur Wehr setzen muss.

Das emotionale Auftauen vollzieht sich in Amerika zweigleisig: in der Relation zu dem Doktorvater sowie in der Liebe. Der Betreuer wird zur wichtigsten männlichen Bezugsperson, zur Vaterfigur, die Hainbachs berufliche Festlegung nachhaltig prägt: „Es war wichtig für mich, ihn nicht zu enttäuschen.“ (THOME 2012:211) Doch je besser er den Professor und die Dimensionen von dessen persönlicher Tragödie kennenlernt, desto mehr evolviert das MeisterSchüler-Verhältnis in Richtung Vertrautheit und Freundschaft. Bei der gemeinsamen Archivarbeit an der Klärung der Todesumstände des im Zweiten Weltkrieg in Deutschland gefallenen Bruders erblickt Hainbach hinter der wortkargen Fassade des alten Mannes Emotionen, die er nie vermutet hätte. Allmählich wird ihm deutlich, dass die sachliche Kälte letztlich nichts weiter als die Flucht vor Gewissensbissen bedeutet und aus Notwehr geboren ist; dass es bei der Kälte um das Management eines ganz bestimmtes Gefühls geht, nämlich um die Angst, als Mann versagt zu haben. Die Erfahrung, im Habitus der kühlen Sachlichkeit manifestiere sich der Wunsch nach Panzerung der eigenen Verletzlichkeit, ist dabei hilfreich, um die eigene Panzerung in der Liebesbeziehung zu Sandrine abzulegen, einer emanzipierten Französin, die dem Zurückgezogenen beibringt, was es bedeutet, das Leben in vollen Zügen zu genießen. Nach der Rückkehr gerät der Mann erneut in verfahrene Muster der Reserviertheit. Die partnerschaftlichen Relationen, die er auf die deutsche Unipraxis überträgt, bringen ihm zwar die Sympathie der Studenten ein, vonseiten der Kollegen erntet er aber vorwiegend Spott. Eine viel größere Enttäuschung hat er jedoch im Privaten einzustecken: Sandrine lässt 
lange auf sich warten, kommt aber letzten Endes nicht aus Übersee. Nicht, dass Hainbach währenddessen enthaltsam wäre, doch das, was er sucht - die große, leidenschaftliche Liebe unter dem romantischen Vorzeichen , auf ewig dein “-, korrespondiert wenig mit dem Zeitgeist in Kreisen, in denen er zu Beginn der 1980er Jahre in Berlin verkehrt. „Im Grunde tickst du wie die frommen Bauern in dem Kaff, aus dem du kommst. Für dich bin ich bloß ein notgeiles Miststück.“ (THOME 2012:176) Dies wirft ihm eine verheiratete Geliebte vor, als er, der Gefühlsarmut überdrüssig, aus der Ménage-à-trois ausbricht.

Mit dem Männlichkeitsbild, das der experimentierfreudige Ehemann zur Schau stellt, kann Hainbach in der Tat wenig anfangen. Diese Zwischenstation, wie die übrigen auch, zeichnet Thome mit wenigen Strichen, gleichwohl zeigt sich der Nebenbuhler mit seiner stolzen Selbstdefinition „ich assoziiere Bärte immer mit Männlichkeit“ (THOME 2012:174) unverkennbar als ein Kulturrevolutionär von einst, freilich in der Version des „Macho als mürrischer Veteran“ (REIMANN 2010:244). Vom kulturrevolutionären Männlichkeitsideal bleibt das äußere Erscheinungsbild erhalten - die „gezielte, protestorientierte Verwahrlosung und Ironisierung des bürgerlichen Anstandsideals“, die mit „körperlichen Zeichen subversiver Männlichkeit, wie zum Beispiel dem bohemehaften Bartwuchs und einem Habitus, den man später als ,cool' bezeichnen würde" (REIMANN 2010:239), verbunden ist. Hinter habituellen Ausdrucksformen wie der rebellischen Antifrisur - dem einstigen Sinnbild unverbrauchter Natürlichkeit und sexueller Allmacht, welche die konventionelle Welt der systemkonformen und in ihrem Intimleben depravierten Bürger herausfordern sollte - verbergen sich keine Gesten maskuliner Unbeugsamkeit oder Machtansprüche mehr, sondern Verzweiflung und Resignation, jene ,"linke Melancholie, mit der die Frustration des kulturrevolutionären Machismo bezeichnet“ wird (REIMANN 2010:244). In die Küchenschürze eingewickelt, sieht der Mann nicht aus, als würde er ,gleich aufspringen und die Revolution ausrufen": „Statt den Sturm auf die Bastille anzuführen, fragt Klaus nach dem Zustand der Klöße [...].“ (THOME 2012:171) Der Schwerfälligkeit des (einvernehmlich) betrogenen Ehemanns sieht Hainbach mit gemischten Gefühlen zu: Er „kann sich nicht entscheiden, ob er $\mathrm{Zu}$ - oder Abneigung empfindet, Achtung oder Mitleid“, merkt aber, dass die Atmosphäre einer Befreiung aus geschlechter- und körperpolitischen Normen seinem Gegenüber nur noch Depressionen statt Inspiration verschafft. Dabei gilt es, die innere Müdigkeit durch Ironie zu kompensieren, um weiteren Enttäuschungen vorzubeugen. Wohin der Protagonist auch blickt, es stehen ihm entweder traurige oder abschreckende Ruinen der Männlichkeit 
Joanna Drynda

vor Augen, wie bei den älteren Bonner Universitätskollegen - ,[u]nglaublich elitäre Typen“ (THOME 2012:213) -, die, hinter starren Formeln und Zeremonien verschanzt, ihn spüren lassen, dass er ihrer Vorstellung von akademischer Kultiviertheit nicht entspricht. „Ich hab sie gehasst. Richtig gehasst“ (THOME 2012:213), gesteht Hainbach rückblickend. Hass bedeute freilich, so VOLKER CAYSA (2008:38), immer Lust am Kampf: „Wer echt hasst, der beobachtet sein Gegenüber überscharf [...], um die Gefahr zu beseitigen und selbst dadurch nicht nur über-, sondern auch aufzuleben." Obwohl der Hass auf selbstgerechte Machtansprüche der Kollegen Hainbach zum Aufstieg anspornt, wird er trotz aller Erfolge das Gefühl nicht los, nicht dazuzugehören, was eine Abschirmung gegen eventuelle Niederlagen notwendig macht: „[W]as ich ausübe, ist in erster Linie ein Beruf. Er ernährt eine Familie.“ (Thome 2012:211) Zudem merkt man, wie sehr der Mann von der hohen Gefühlsintensität überrascht ist und sich bemüht, seinen Hass wegzurationalisieren. Dabei sei dieses Gefühl, so CAYSA (2008:39) weiter, nicht einfach widervernünftig. Ganz im Gegenteil: „Die analytische Kraft des echten Hassenden zerlegt seine Gegner ohne Schmerzen.“ Die Fähigkeit, die Männerbilder um ihn herum rational zu zerlegen, hilft dem Protagonisten kaum, das eigene Konzept fest aufzubauen. Vielmehr wird dieses aus Versatzstücken konstruiert, mal um sich von der jeweiligen Männerwelt abzugrenzen, mal um einen Anschluss zu ihr zu finden: ,[...] über seinen eigenen Schatten springen - Variation einer Disziplin, in der er seit Jahren trainiert und eine gewisse Routine ausgebildet hat" (THOME 2012:360). Unabhängig davon, wie stark der Mimikry-Wunsch ist, wird der Protagonist wie bei der Fliehkraft stets nach außen weggeschleudert, in eine Outsider-Position, die Widersprüchliches in sich vereint: Als „Achtundsechziger“ (THOME 2012:353) hängt er an bürgerlichen Normen; als Romantiker, der wider den Zeitgeist in der Verbindlichkeit eine Tugend erkennt, betrügt er die geliebte Ehefrau gelegentlich mit Sandrine; als Liberaler, der Frauenemanzipation befürwortet, blüht er in alten Rollenzuweisungen auf; als einer, der im Privaten Emotionen entdeckt, etwa die Zärtlichkeit als Vater, ordnet er sich im Beruflichen hegemonialen Vorstellungen von Männlichkeit unter. Je mehr sich der Mann bemüht, die Diskrepanzen zusammenzudenken, desto weniger klappt es: „Es ist, wie Bernhard gesagt hat: Bei anderen sieht es leicht aus. Nicht zu viel nachdenken. Einfach leben.“ (THOME 2012:297) Anders als Marie vermutet, beruht Hainbachs Ich-Konstrukt nicht auf einer strengen Gefühlskontrolle, die dazu auffordert, Emotionen so zu managen, dass sie nicht an der Oberfläche auftauchen oder - besser noch - überhaupt entstehen. Der äußeren Kühle steht eine innere Leidenschaft gegenüber, für die der Protagonist nur selten 
einen passenden Ausdruck findet. Bei dem Rationalitätschild geht es also weniger um die Abwehr des Gefühls, denn um die Abwehr der Gefahr, sich als Mann in seiner Unzulänglichkeit bloßzustellen.

\section{4. ,weil gerade an Grenzgang niemand wusste, wo die Grenze eigentlich verlief" (THOME 2009:227)}

Die Probleme mit dem Abstecken der Männlichkeitsgrenzen sind dem Protagonisten in Grenzgang bekannt: „Sei ein Mann. So hatte es Konstanze ausgerückt.“ (ThOME 2009:39) „Sei ein Mann, hatte sie gesagt, und nun würde er versuchen einer zu sein - bloß vielleicht ein anderer, als sie wünschte." (THOME 2009:55) Nahezu obsessiv erinnert sich Thomas Weidmann an die dringlichen Appelle der Freundin, ohne dahinterzukommen, wie das ,MannSein' konturiert sein sollte. Fest steht nur, dass sich seine und ihre Auffassung davon kaum decken: „Sei ein Mann. [...] Du musst dich nicht verstellen. Sei sauer, traurig, frustriert, du hast allen Grund dazu. Aber erlaub der Sache nicht, dein Leben zu zerstören.“ (THOME 2009:56f.) Den Anlass zum Streit über die Männlichkeit bieten einerseits der Umgang mit dem Karriereknick, andererseits Weidmanns Vorstellungen von der gemeinsamen $\mathrm{Zu}$ kunft. Die Enttäuschung, dass die Berufswahl fehlschlug, die nicht der „Zufall, sondern eine Leidenschaft für die Sache bestimmt hatte" (THOME 2009:78), ist zu tief, als dass sie sich verbergen ließe, doch weigert sich der Mann beharrlich, sie sowohl vom Gesamtkonzept des Lebens abzukoppeln als auch den Schmerzgefühlen Ausdruck zu geben. Stattdessen wird „das unabweisbare Bewusstsein seiner eigenen Jämmerlichkeit“" (THOME 2009:55) der Freundin gegenüber mit wortkarger Kälte oder mit Zynismus kaschiert: „Er hatte sich unter Kontrolle, immer noch, er wurde sarkastisch, ihm lagen Gemeinheiten auf der Zunge, aber wütend wurde er nicht.“ (THOME 2009:58) Die starke Identifikation mit dem Beruf deutet darauf hin, dass die (wissenschaftliche) Arbeit als die einzige feste Koordinate in Weidmanns Selbstbild fungiert: „Er war nicht der Typ fürs Provisorium. Er führte Buch über Bücher, die er las.“ (THOME 2009:73) Umso größer sind die Verwüstungen, die der Verlust der Forschungsstelle in seinem Selbstverständnis als Mann anrichtet. Doch beschränken sich Plädoyers für dauerhafte Strukturen ausschließlich auf den beruflichen Bereich, im Privaten wird jede Verbindlichkeit vermieden: „Konstanze nannte es sein Distanz-Syndrom und arbeitete auf ihre Weise daran, es ihm auszutreiben [...].“ (THOME 2009:386) Je öfter sie vom Zusammenziehen und vom Kinderwunsch spricht, desto einfallsrei- 
cher werden die Ausreden. „Niemand, der heiratet, entgeht der Banalität der Ehe“ (THOME 2009:168), verkündet der Mann und ,stellt sich auch ehelichen Sex öde vor: Müde, missionarisch, monatlich. Beim Stichwort Familie nahm er alle Stereotype für bare Münze [...].“ (THOME 2009:77)

Vor der würgenden Angst, als Mann rundweg versagt zu haben, rettet sich der Protagonist durch die Flucht. Suchte er bislang vergebens nach einem Funken Wut, der groß genug wäre, um ihn in Flammen zu setzen und zu Taten zu treiben, so erfüllt ihn wilde Wut, als er seine Sachen aus dem Büro räumt: ,[...] das erste echte Gefühl seit langem; eins, das er nicht mit Gedanken aus sich herauskitzeln musste, um es zu empfinden“ (THOME 2009:41). Das vorgetäuschte Mitgefühl, gepaart mit Schadenfreude und dem Triumph der Kollegen, als Sieger davonzukommen, bringt beim Abschied den Stein ins Rollen, und zwar buchstäblich. Das Ausmaß der in fremden Augen lesbaren Versagung übersteigt die Bewältigungskapazität des Protagonisten. Ganz außer sich, greift er die angebliche Ursache der Versagung an, indem er einen Stein in das Institutsfenster schleudert. Danach fährt er überstürzt davon weg von Berlin, von der Universität und obendrein von Konstanze. „Mag Wut auch eine selbstsuggestive Wirkung haben, die Stärke verleiht", so RoLF HAUBL (2008:25) über Wut als einen aggressiven Leitaffekt, „dann bewirkt sie das doch nur vorübergehend und um den Preis eines mehr oder weniger gravierenden Realitätsverlusts." Auf Weidmann trifft dies insofern zu, als er amokartig die Heimatstadt ansteuert, die ihm wegen der Tristesse und Perspektivlosigkeit seit jeher zuwider war.

Bei der Ankunft bewegen ihn widerstrebende Gefühle: „Er war alles gleichzeitig: aufgekratzt, erschöpft, gelangweilt, gespannt, nervös.“ (THOME 2009:128) Mit seinem inneren Grenzerlebnis fügt sich der Mann in die Atmosphäre der Stadt ein, in der gerade Grenzgang gefeiert wird. Ursprung und Zweck des mit großem Pomp zelebrierten Festes ist das Ablaufen der einst mit Steinen markierten Ortsgrenzen. Dem alten Brauch entsprechend, mit Requisiten aus ferner Vergangenheit ausgestattet, gehen die Bewohner gemeinschaftlich die Grenzmarkung ab, um eventuelle Verschiebungen zu überprüfen und nebenbei auf steinigen Waldwegen ihre eigenen Grenzen zu testen. Aus Lautverstärkern dröhnen dabei feierliche Ansprachen, die den Grenzgang als ,eine Tradition [preisen], in der sich die Verbundenheit ausdrückt zwischen gestern, heute und morgen, zwischen den Generationen“, als „die Feier all dessen, [...] worauf wir stolz sind, was uns die Gewissheit gibt, dass wir Mitglieder einer Gemeinschaft sind, in der Mitglied zu sein sich lohnt“ (THOME 2009:122f.). 
Die Rede des Bürgermeisters hat eine symbolische Bedeutung. Einerseits werden nach den Leitgedanken die Romanteile benannt (Der Stein..., ...die Grenze..., ....in Ewigkeit). Außer dass die Stichworte metaphorisch zu den Festtagen in Beziehung stehen, um die alle Reminiszenzen aus dem Zeitraum von insgesamt achtundzwanzig Jahren (1985-2013) kreisen, markieren sie andererseits den Bewegungsradius der Figuren, die innerhalb der strengen geographischen wie mentalen - Grenzen herumlaufen, ohne den engen Zirkel anders als nur flüchtig zu verlassen. Auf der Metaebene wird in der Rede eine Diagnose über moderne Befindlichkeit vorweggenommen, freilich in Form eines Wunschbildes, das schwer erreichbar ist, besonders für denjenigen, die wie Thomas Weidmann oder Kerstin Werner nicht nur tief in der Provinz, sondern auch in einer Midlifecrisis stecken: „Wir sind Mitte vierzig, und das Leben läuft an uns vorbei.“ (THOME 2009:218) Beide im Hinterland gestrandeten Großstädter haben schwer an ihrem Provinzleben zu tragen, das keine Illusionen zulässt, weil es über den Alltag nicht hinausweist und selten eine nennenswerte Ablenkung bietet: „Zeit vergeht, und nichts passiert [...].“ (THOME 2009:161) Außer den nichterfüllten Hoffnungen, der Vertrautheit mit Enttäuschungen sowie der Art, wie die zwei Verlierer versuchen, den Umständen zum Trotz ihre angetastete Würde und ihren Stolz zu wahren, gibt es zwischen ihren Geschichten keinerlei Berührungspunkte. Denn die Frau blickt auf wenig aufregende Erlebnisse zurück, die nahezu wortgetreu die Befürchtungen des Mannes bestätigen, „dass alle Ehen in der Form eines Klischees enden, wenn sie denn enden“ (THOME 2009:216): „Prioritäten verschieben sich, aus Liebe wird Routine, aus Routine Langeweile, aus Langeweile Streit.“ (THOME 2009:215) Einen vergleichbar spektakulären Steinwurf wie den von Weidmann hat Kerstin Werner nicht zu verzeichnen, vielmehr war sie darum bemüht, als Hausfrau dem Mann alle Steine aus dem Wege zu räumen, um ihm einen Karrieresprung zu ermöglichen. Nach zehn Jahren ist der Traum von einem festen Platz im Leben definitiv zerplatzt, über den Prinzen von einst, der nun seine „vom Familienleben angekratzte Männlichkeit“ (THOME 2009:114) durch die Anschaffung von Motorrädern und durch eine jüngere Frau aufrichtet, äußert sie sich trocken: „Ein Mann, ein Wort, breite Schultern, ein Schwanz. [...] Mit anderen Worten: Ein Arschloch [...].“ (THOME 2009:95)

Über Jahre hinweg kreuzen sich die Wege der zwei Hauptfiguren, bevor sie zaghaft zueinander finden. Beide führen ein Leben, dass sie nie gewollt haben und über das sie sich fragen, ob es nicht stehengeblieben ist auf halber Höhe, ob sie sich im Aufstieg oder im Abstieg befinden. Zum einen wissen sie um alle Schritte, die offenbar getan wurden, um diejenigen zu unterlassen, 
die hätten getan werden müssen, und reflektieren in Gesprächen den eigenen Beitrag zu der persönlichen Misere:

Ihnen stößt etwas zu, und statt sich dagegen zu stemmen, geben Sie der Veränderung nach, folgen ihr noch ein Stück weiter, als Sie gezwungen worden sind. Letztlich ein Versuch, die Hoheit über das Geschehen zurückzugewinnen, weil Sie am Ende an einem Punkt landen, zu dem Sie aus freien Stücken gelangt sind. (THOME 2009:188)

Zum anderen wollen sie nicht aus der Apathie erwachen, denn das lethargische Dahinleben - ein immanent widersprüchlicher Zustand - entbehrt jeder emotionalen Aufruhr, die womöglich weitere Schmerzen zufügen könnte: „das Verschwinden der Sehnsucht und der Eintritt in eine Art Leere, [erfüllt] einen mit viel weniger Angst [...], wenn mal erst drin ist“ (THOME 2009:21). Dennoch finden sie es irritierend, „unter etwas zu leiden, das keinen Schmerz verursacht. Nur ein winziges Ziehen, ein sanfter Druck, der sich kaum lokalisieren lässt [...].“ (THOME 2009:160) Von Einsamkeit geplagt, spüren beide intuitiv, dass sie viel zu lange im Kreis gelaufen sind und dass der Teufelskreis der Flucht - durch das wiederkehrende Grenzgangfest versinnbildlicht dann zu durchbrechen wäre, wenn sich Grundmuster ändern, nach denen sie handeln. Aus Gewohnheiten auszubrechen und jenseits der Vierzig eine neue Qualität an sich zu entdecken, bedeutet für sie etwas anderes. Die Frau hat sich der Herausforderung zu stellen, fremde Erwartungen zu ignorieren, die sie auch um den Preis der Demütigung mit dem obligaten Lächeln mustergültig erfüllt. Der Traum, „das abgetragene Leben“ (THOME 2009:78) im biographischen Sinn aufzufrischen, hat für den Mann schon deshalb eine andere Dimension, weil seine früheren Wünsche auf den ersten Blick paradoxerweise in Erfüllung gingen; die Arbeit hat den höchsten Stellenwert in dem Tagesablauf, auch wird die Freiheit von keiner familiären Verpflichtung eingeschränkt. Trotzdem fällt die Bilanz aus der Provinz- und der anbrechenden Altersperspektive ernüchternd aus: „Jahrelang hat er versucht, mehr zu sein, als er war“ (THOME 2009:283), da er ,nie Ehrgeiz im eigentlichen Sinne besessen hatte, sondern allenfalls dessen hässlichen Zwilling, die Eitelkeit“ (THome 2009:137). War der Protagonist Weidmann selbst einer der erfolgshungrigen Männer, „die an ihrem aufgeblasenen Ego hingen wie an einem Heißluftballon ohne Gondel: Zappelnd, grotesk, vom Absturz bedroht" (THOME 2009: 283), so lernt er langsam schätzen, dass die Leute um ihn herum, anders als die Großstädter, nicht unbedingt das Beste aus ihrem Leben machen wollen, sondern sich - pragmatisch im Leben - mit dem Guten zufrieden geben. 
Doch ist es keine Pragmatik, die den Mann erst in die Arme von Kerstin Werner und dann zum Standesamt führt, sondern Liebe. Dem leise aufkeimenden Gefühl blickt er zunächst äußerst skeptisch entgegen, da er nicht sicher ist, ob er gerade dieser Frau gegenüber zu einer Nicht-Verstellung fähig wäre. Denn nach dem Bruch mit Konstanze glaubt er, keine Begabung für Beziehungen zu haben und perfektioniert über Jahre hinweg die Fähigkeit, keine Missverständnisse aufkommen zu lassen und sich nicht zu verlieben, sondern ein aufmerksamer, sprungbereiter und unsentimentaler ,Spieler zu sein“ (THOME 2009:322), denn ,zu dauerhafter Enthaltsamkeit fühlt er sich nicht berufen“ (THOME 2009:231). Nach der Anmeldung in einem Dating-Portal lebt er seine Jagdinstinkte durch flüchtige Bekanntschaften aus, um sich abzulenken und die kurze Spannung zu genießen, wenn auch hin und wieder die lange Enttäuschung danach mit einem hochprozentigen Getränk heruntergespült werden muss. Da er dabei ,an zu vielen Frauen seines Alters die Folgen dauernden Alleinseins beobachtet, all diese Ticks, Ängste und Zwänge, das zu schrille Lachen, die ins Taschentuch geschnäuzte Hysterie eines plötzlichen Weinkrampfs“ (THOME 2009:234), erfasst ihn Panik angesichts der offen gezeigten Zuneigung der Frau, die er als Überrumpelung empfindet:

Und jetzt sieht er zu, wie Kerstin Werner ihm diese Schlinge um den Hals legt, als ob er vorhätte, auf seine alten Tage zum Kleinstadt-Kantianer zu mutieren: Handle so, dass die Maxime deines Willens jederzeit als Prinzip einer langweiligen Ehe gelten könne.“ (Тноме 2009:323)

In der Geschichte der spätsommerlichen Liebe veranschaulicht Thome eindrucksvoll „,eine kulturelle Neukodifizierung von Geschlechterungleichheiten“ (ILlOUZ 211:120) und macht die spätmoderne Asymmetrie zwischen Männern und Frauen genau an dem Punkt fest, an dem ,[s]tabile Intimbeziehungen zumal für Frauen schwer auf die Beine zu stellen sind, weil die Männer emotional ausweichen und regelmäßig den Versuchen der Frauen widerstehen, sich langfristig zu binden.“ (ILLOUZ 2011:130) Der Emotionsforscherin zufolge benachteilige die heutige Situation die Frauen schon deswegen, weil für sie die Partnerwahl in einem von der sexuellen Attraktivität begrenzten Zeitraum stattfinde. Mit dieser Zeitwahrnehmung gehe besonders bei Frauen in ihren Vierzigern der Eindruck schwindender Optionen einher (vgl. ILlOUZ 211:149). Kerstin Werner ,spürt förmlich, wie ihr die Zeit davonläuft" (THOME 2009:327), was ihre Herzenswünsche und Erwartungen an den Mann immer bescheidener werden lässt: „Nur da zu sein hätte er, physisch, männlich, beidhändig; einer, den man irgendwann ohne Hintergedanken vorschlagen kann, ins Bett zu gehen. Weiter geht sie selbst in Gedanken nur 
Joanna Drynda

selten.“ (THOME 2009:107) Im Unterschied dazu müssen Männer derzeit weder mächtig noch dominant sein, um sexuellen Zugang zu Frauen zu erlangen, was dazu führt, dass die serielle oder kumulative Sexualität mit einem männlichen Statusmerkmal assoziiert wird (vgl. ILLOUZ 211:142). Demzufolge äußert sich die moderne Männlichkeit eher in einer emotionalen Verweigerung als darin, Gefühle unter Beweis zu stellen: „Emotionale Distanziertheit ließe sich als Metapher für eine männliche Autonomie verstehen, die mit der Trennung von Sex und Ehe nur noch realisierbarer wurde." (ILLOUZ 2011:143)

Vor diesem Hintergrund ist Thomas Weidmann ganz ein Kind seiner Zeit. Wie Illouz in Bezug auf die Theorie des Historikers John Tosh konstatiert, hat die traditionelle dreigleisige Männlichkeitsstruktur (Autorität im Haushalt, Unabhängigkeit am Arbeitsplatz, gegenseitige männliche Solidarität) im Laufe des 20. Jahrhunderts durchgängig ihre Gültigkeit verloren (vgl. ILLOUZ 211:142). Zudem wurde die Etablierung der zentralen Rolle der Sexualität in der Neudefinition von Männlichkeit im Zuge der 68er Bewegung wesentlich beschleunigt, die alle Intimbeziehungen aus einem moralischen Rahmen löste. Da die antibürgerliche Revolte, so FREVERT (2010:326f.), bestimmte emotionale Modi der In- und Exlusion wiederholte, generierten die neuen Gefühlstandards einen hohen Konformitäts- und Anpassungsdruck: „Wer sich den kollektiven Lockerungsübungen nicht unterwarf, wer die Formen angeblich befreiter Sexualität nicht schätzte und nach wie vor ,bürgerliche“ Gefühle hegte, der hatte es schwer", akzeptiert zu werden. Es dauert lange, bis der Protagonist seine Liebesbedürftigkeit zugibt und die routinierte Maske des coolen Don Juan ablegt, ohne befürchten zu müssen, damit gegen die Grenze der Männlichkeit zu verstoßen.

\section{Fazit: ,,... das will ich festhalten. Die Veränderung. Die sich übrigens nicht festhalten lässt, aber es lohnt den Versuch“" (THOME 2012:143)}

Der Satz, mit dem der Protagonist in Fliehkräfte erklärt, warum er ein Tagebuch führte, lässt sich als das poetologische Credo des Autors interpretieren. Denn Thome schildert in seinem Romanwerk nicht nur die Transformationen gesellschaftlicher Ideale, kultureller Werte und normativer Gefilde, sondern versucht auch einzufangen, in welchem Maße die Veränderungsprozesse die konstitutive Semantik moderner Subjektivität prägen. Die Kombination von Gesellschaftspanorama mit der gleichzeitigen Konzentration auf das Detail 
Zu (männlichen) Ausbrüchen aus emotionaler Routine im Romanwerk von ...

lässt den Autor die Schlüsselerlebnisse einer Gesellschaft ausbuchstabieren: Die erzählten Geschichten führen zum einen viele der Aporien vor Augen, die die Beziehungen zwischen Männern und Frauen in der Spätmoderne kennzeichnen sowie die Desorientierung der Menschen angesichts der vom Arbeitsmarkt geforderten sozialen Mobilität und des Leistungsdrucks. Zum anderen schwingt darin ein ausführliches Emotionsregister des verunsicherten Individuums mit. Den Kristallisationspunkt der teils melancholischen, teils witzig pointierten Narrationen bilden die verzweifelten, mehr oder minder geglückten Versuche männlicher Protagonisten, sich aus emotionaler Routine zu befreien, die in Zeiten des Abgesangs auf habituelle Männlichkeitsnormen, in unterschiedlicher Ausprägung dem männlichen Subjekt als ein notdürftiger Schutzbau für "sein geschundenes Ego“ (THOME 2009:172) dient.

\section{Literatur}

Berninger, Anja / Döring, SABine A. (2009): Einleitung. In: Döring, 141-147.

Borutta, Manuel / Verheyen, Nina (eds.) (2010): Die Präsenz der Gefühle. Männlichkeit und Emotion in der Moderne. Bielefeld.

Borutta, Manuel / Verheyen, Nina (2010): Vulkaniker und Choleriker? Männlichkeit und Emotion in der deutschen Geschichte 1800-2000. In: BORUTTA / VERHEYEN, 11-39.

CAYSA, VOLKER (2008): Der Hass - eine große Stimmung. In: UHLIG, STEPHAN (ed.): Was ist Hass? Phänomenologische, philosophische und sozialwissenschaftliche Studien. Berlin, 35-48.

Damasio, Antonio R. (2000): Ich fühle, also bin ich. Die Entschlüsselung des Bewusstseins. Aus dem Englischen von Hainer Kober. München.

Döring, SABINE A. (ed.) (2009): Philosophie der Gefühle. Frankfurt (M.).

- (2009): Allgemeine Einleitung: Philosophie der Gefühle heute. In: DÖRING, 12-65.

ELLMEIER, ANDREa / INGRISCH, Doris / WALKENSTEINER-PrESCHL, ClAUdia (2012): Zur Einleitung. In: EllmeIER, ANDreA / INGRISCH, DoRIS / WALKENSTEINERPreschl, Claudia (eds.): Kultur der Gefühle. Wissen und Geschlecht in Musik, Theater, Film. Wien/Köln/Weimar, 7-21.

Frevert, Ute (2010): Gefühlvolle Männlichkeiten. Eine historische Skizze. In: BORUTTA / VERHEYEN, 305-330.

Goldie, PeTER (2009): Emotionen und Gefühle. Übersetzt von Jean Moritz Müller. In: DÖRING, 369-397. 
Joanna Drynda

Haubl, Rolf (2008): Ärger, Wut, Zorn - Hass. In: Uhlig, StePhan (ed.): Was ist Hass? Phänomenologische, philosophische und sozialwissenschaftliche Studien. Berlin, 23-27.

IllouZ, Eva (2012): Warum Liebe weh tut. Eine soziologische Erklärung. Aus dem Englischen von Michael Adrian. Berlin.

- (2013): Die neue Liebesordnung. Frauen, Männer und „Shades of Grey“. Aus dem Englischen von Michael Adrian. Berlin.

RECKWITZ, ANDREAS (2010): Umkämpfte Maskulinität. Zur Historischen Kultursoziologie männlicher Subjektformen und ihrer Affektivität vom Zeitalter der Empfindsamkeit bis zur Postmoderne. In: BORUTTA / VERHEYEN, 57-77.

ReIMANn, ArIBERT (2010): Zwischen Machismo und Coolness. Männlichkeit und Emotion in der westdeutschen ,Kulturrevolution' der 1960er- und 1970er-Jahre. In: BORUTTA / VERHEYEN, 229-253.

ROBERTS, ROBERT C. (2009): Was eine Emotion ist: eine Skizze. Übersetzt von Christoph Ammann. In: DöRING, 169-201.

- (2009a): Emotionen. Ein Essay zur Unterstützung der Moralpsychologie. Übersetzt von Christoph Ammann. In: DöRING, 269-292.

SCHOLZ, SYlKa (2010): Vom starken Helden zum zärtlichen Vater? Männlichkeit und Emotionalität in der DDR. In: BORUTTA / VERHEYEN, 203-228.

THOMe, Stephan (2009): Grenzgang. Roman. Frankfurt (M.).

- (2012): Fliehkräfte. Roman. Berlin. 